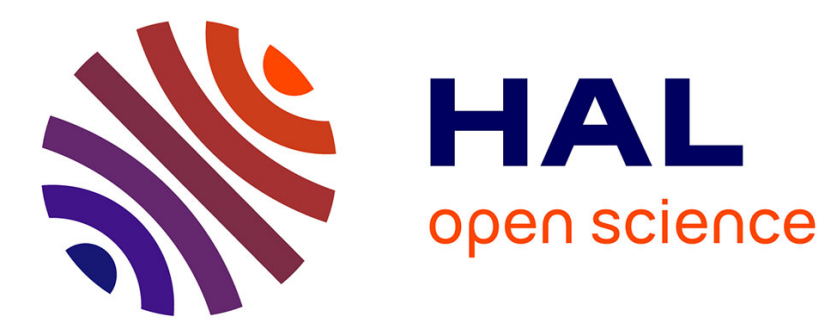

\title{
Heteros theos comme approche du dualisme dans la pensée d'Origene
}

\author{
Izabela Jurasz
}

\section{To cite this version:}

Izabela Jurasz. Heteros theos comme approche du dualisme dans la pensée d'Origene. Chôra. Revue d'études anciennes et médiévales, 2015, Dualismes: Doctrines religieuses et traditions philosophiques, 13 (Supp), pp.465-490. 10.5840/chora20151326 . hal-02550601

\section{HAL Id: hal-02550601 https://hal.sorbonne-universite.fr/hal-02550601}

Submitted on 22 Apr 2020

HAL is a multi-disciplinary open access archive for the deposit and dissemination of scientific research documents, whether they are published or not. The documents may come from teaching and research institutions in France or abroad, or from public or private research centers.
L'archive ouverte pluridisciplinaire HAL, est destinée au dépôt et à la diffusion de documents scientifiques de niveau recherche, publiés ou non, émanant des établissements d'enseignement et de recherche français ou étrangers, des laboratoires publics ou privés. 
$\chi \omega \dot{\rho} \rho \cdot \cdot R E A M$, Dualismes, p. 465-490

\title{
HETEROS THEOS COMME APPROCHE DU DUALISME DANS LA PENSÉE D'ORIGÈNE
}

\author{
Izabela Jurasz \\ (Université Paris-Sorbonne, Centre Léon Robin)
}

\begin{abstract}
Of all the approaches to the concept of dualism in Origen's thought, this article concerns the issue which does not seem very obvious: his understanding of the expression heteros theos. Is it possible to call Jesus "the other God", existing aside from the only God? Thanks to the Dialogue with Heraclides, we can judge the profundity of the issue. According to the Dialogue, heteros theos resembles the language used by Marcion, in which "the other God" means the Demiurge, an antagonist of the merciful God. But the expression also invokes the Judeo-Christian inspired Christology, in which "the other God" signifies a manifestation of the God Jehovah, or a secondary deity, subordinate to Jehovah. In his other writings, Origen usually avoids calling Christ heteros theos, precisely because of the similarity to marcionism and monarchianism. However, forced to resolve the theological problem presented in the Dialogue, Origen decides to explain the meaning of the term "heteros". His explanations are inspired by Aristotle's categories, much simplified and illustrated by examples from the Bible. Origen shows that "difference" can be understood as opposition, but there are other possible interpretations of the term. More than anything, it is relative towards a particular characteristic. Origen's argumentation, in comparison with other discussions concerning the term (Justin Martyr and Tryphon, Peter and Simon the Magician), illustrates that the understanding of God as "different" may lead to the emergence of dualistic concepts, which are often very radical.
\end{abstract}

Parmi les très nombreuses études de la pensée origénienne, celles consacrées à la question du dualisme (quel que soit le contenu attribué à ce terme) restent peu nombreuses. Compte tenu de l'inspiration platonicienne de la pensée d'Origène, certains y voient une source possible du dualisme (U. Bianchi) ${ }^{1}$,

1. Il convient de noter que la définition du dualisme donnée par U. Bianchi est particulièrement large: $c f$. U. Bianchi, «Presupposti platonici e dualistici di Origene, De 
tandis que certains autres l'attribuent à la polémique anti-gnostique, principalement anti-marcionite (P. O'Cleirigh, C.A. Spada) ${ }^{2}$. Certes, Origène est un polémiste anti-gnostique des plus originaux et il n'est pas facile de préciser en quoi consiste cette «influence» gnostique, car Origène tout en critiquant les doctrines marcionites et valentiniennes, n'est pas indifférent à la problématique qu'elles soulèvent et n'hésite pas à s'approprier certains concepts - quitte à leur donner une interprétation propre. L'exemple que nous avons choisi permet d'approcher cette façon originale dont Origène aborde la question centrale du christianisme: celle de l'unité de Dieu, à l'épreuve de l'affirmation de la divinité de Jésus-Christ. Telle est la question examinée dans l'Entretien avec Héraclide (rédigé dans les années 244-249) ${ }^{3}$, dans lequel Origène appelle

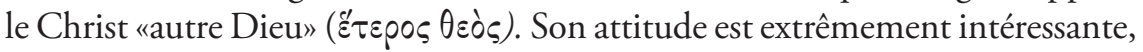
car il s'agit d'une expression communément associée à la doctrine marcionite et spontanément interprétée par ses interlocuteurs en un sens dualiste.

\section{L'Entretien avec Héraclide et position du problème}

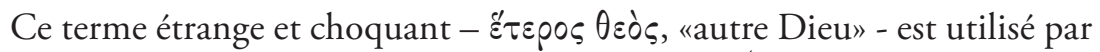
Origène pendant le débat avec les évêques d'Arabie ${ }^{4}$, conservé sous le titre Entretien avec Héraclide. Le texte fournit peu d'informations sur le lieu exact et la date du débat, mais le ton de la conversation permet néanmoins de conclure qu'Origène était déjà venu dans cette province et qu'il avait l'habitude

Principiis», dans H. Crouzel, A. Quacquarelli (dir.), Origeniana Secunda. Second colloque international des études origéniennes (Bari, 20-23 septembre 1977), Roma, Edizioni dell'Ateneo, coll. "Quaderni di Vetera Christianorum», n 15, 1980, p. 37. Les études du platonisme chez Origène sont beaucoup plus nombreuses, mais la question du dualisme n'est pas toujours placée au centre.

2. P. O'Cleirigh, "The Dualism of Origen», dans R.J. Daly (dir.), Origeniana Quinta. Papers of the 5th international Origen congress, Boston college, 14-18 August 1989, Leuven, University Press, coll. «Bibliotheca ephemeridum theologicarum Lovaniensium» (désormais abrégée en BETL), n 105, 1992, p. 346-350. Aussi J. Rius-Camps, "Origenes y Marcion. Caracter preferentamente antimarcionita del prefacio y del secundo ciclo del Peri Archon», dans H. Crouzel, G. Lomiento, J. Rius-Camps (dir.), Origeniana. Premier colloque international des études origéniennes (Montserrat, 18-21 septembre 1973), Bari, Istituto di Letteratura Cristiana Antica, coll. "Quaderni di Vetera Christianorum», n 12, 1975, p. 297-312; C.A. Spada, "Aspetti della polemica antimarcionita nel ComJn», dans Origeniana Quinta..., op. cit., p. 85-91.

3. Entretien d'Origène avec Héraclide, introd., texte, trad. et notes J. Scherer, Paris, Cerf, coll. «Sources chrétiennes» (désormais abrégée en SC), n 67, 1960.

4. Il s'agit de la province romaine de l'Arabie (Arabie pétrée) créée en 106 après la conquête du royaume nabatéen. Elle fut un territoire stratégiquement important, situé entre l'égypte, la Judée et la Syrie. La plus importante communauté chrétienne de l'Arabie se trouva dans sa capitale, à Bostra. 
de ce genre d'entretiens ${ }^{5}$. Le texte qui nous est transmis n'est pas un écrit d'Origène lui-même, mais plutôt un compte rendu. Sans trancher sur le moment de la rencontre, l'éditeur français propose de la situer dans les années $243-245^{6}$.

$\mathrm{Au}$ cours de l'entretien, Origène répond à de nombreuses questions théologiques (l'humanité du Christ, l'âme, la résurrection, la prière, etc.), bien que le motif de son invitation en Arabie fut la difficulté posée par l'interprétation d'un passage biblique disant que «l'âme est le sang» (Lév. 17, 11). Or, certains imaginaient que l'âme, après la mort, restait dans le corps, dans le tombeau, réellement morte jusqu'à la résurrection $(10,19-21)$. Dans ce contexte-là, on aborde forcément l'exemple de Jésus, de sa mort et de sa résurrection.

Selon les thématiques abordées, le texte se laisse diviser en trois parties:

- le dialogue d'Origène avec les évêques Héraclide et Maxime (I, 6 - 10, 13) au sujet de la divinité de Jésus, la nature de son corps et la résurrection des âmes.

- l'échange autour de la question posée par l'évêque Denys: l'âme est-elle le sang? $(10,13-24,17)$

- un autre débat provoqué par la remarque de l'évêque Démétrius sur l'immortalité de l'âme $(24,18-28,17)$.

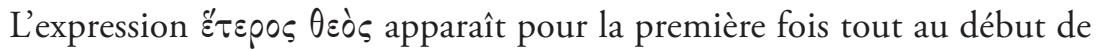
l'Entretien, elle est employée par Origène pour décrire la relation entre Dieu le Père et le Christ. Ce terme met visiblement mal à l'aise l'interlocuteur du théologien alexandrin, l'évêque Héraclide. Il s'ensuit un échange entre ces deux personnages, après quoi Origène fait un discours théologique, extrêmement

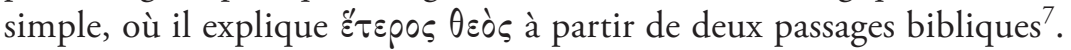

La discussion commence par la confession de foi faite par l'évêque Héraclide, telle qu'on faisait au moment du baptême - la foi en un Dieu unique. Cette confession commence par le texte du Prologue de Jean, les versets 1-3, où il

5. La notice d'Eusèbe de Césarée n'apporte pas de précisions sur les circonstances de l'entretien: "D'autres gens encore, en Arabie, surviennent à l'époque dont nous parlons, introducteurs d'une doctrine étrangère à la vérité. Ils disaient que l'âme humaine, provisoirement dans la conjoncture présente, meurt avec les corps, au moment du trépas, et qu'elle est corrompue avec eux, mais qu'un jour, au temps de la résurrection, elle revivra avec eux. Alors aussi un concile important fut rassemblé. Origène y fut de nouveau convoqué, et, après avoir fait des discours à l'assemblée sur la question discutée, il se comporta de telle manière qu'il changea les pensées de ceux qui avaient d'abord été trompés.» $H E$, VI, 37; éd. Bardy, SC 41, p. 139.

6. L'éditeur propose une datation entre 244-249; cf. Entretien, éd. cit., p. 21-22.

7. Sur le débat autour de l'autre Dieu dans l'Entretien voir M. Simonetti, Studi sulla cristologia del II et III secolo, Roma, Institutum Patristicum Augustinianum, coll. «Studia ephemeridis Augustinianum», n 44, 1993, p. 139-143. L'auteur souligne la grande simplicité de l'argumentation, l'absence de termes techniques et y voit une démarche catéchétique. 
est question du Verbe qui au commencement était auprès de Dieu et par qui tout a été fait. Ensuite, Héraclide résume rapidement les événements fondamentaux de la vie de Jésus et finit par le déclarer «Dieu et homme à la fois». Origène l'interroge à partir de ces déclarations.

La première question posée par Origène concerne le Dieu unique «le tout-puissant, l'incréé, suprême, qui a fait toutes choses». Ayant obtenu l'accord d'Héraclide, le théologien alexandrin revient à la déclaration christologique, mais il la formule en des termes tirés de l'épître aux Philippiens $(2,6)$ où il est question de Jésus-Christ qui, étant en «la forme de Dieu», a pris «la forme de serviteur». La référence au Prologue de l'Évangile selon Jean cité précédemment par Héraclide est à peine perceptible:

Origène dit: Le Christ Jésus existant dans la forme de Dieu, tout en étant autre

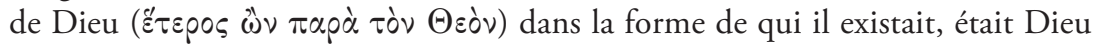
avant d'être venu dans la chair, oui ou non ${ }^{8}$ ?

Origène évoque d'abord le verset de Phil. 2, 6 en parlant explicitement de la "forme de Dieu», tandis que les affirmations du Prologue sont seulement sous-entendues: «ll était Dieu avant d'être venu dans la chair, oui ou non?» Entre ces deux citations ou allusions est glissée l'expression qui fait l'objet du débat:

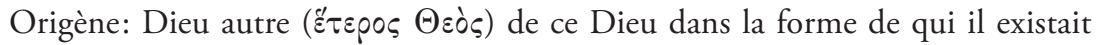
lui-même?

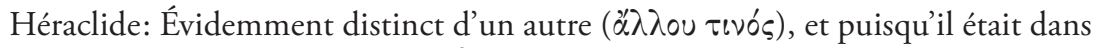
la forme de celui-là, du Créateur?.

Si Héraclide veut respecter les Écritures, il ne peut qu'acquiescer, mais il

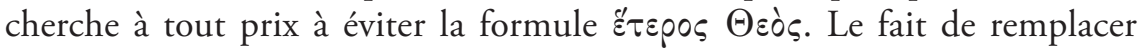

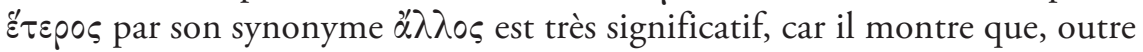
le problème théologique, la formule elle-même pose problème. Héraclide

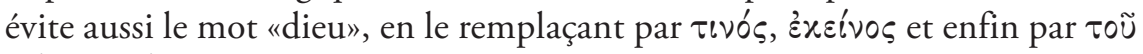

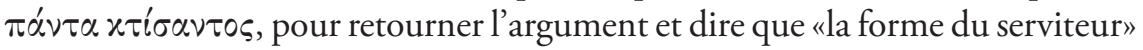

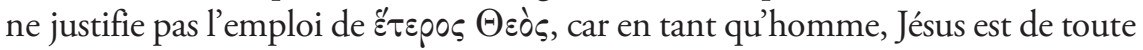
évidence différent de Dieu. Cette réponse oblige Origène à développer autrement son argumentation. Il choisit donc de rappeler un passage de l'épître aux Colossiens en citant l'expression "premier-né de toute la création» $(1,15)$ :

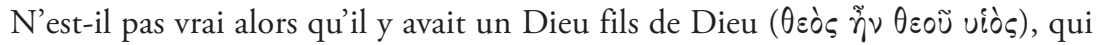

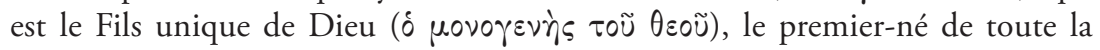

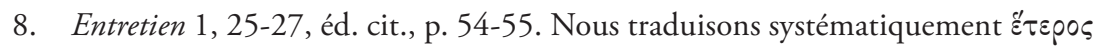
par «autre» tandis que J. Scherer choisi le terme «distinct».

9. Entretien 2, 1-2, éd. cit., p. 54-55. 


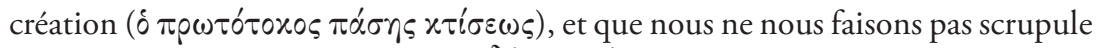

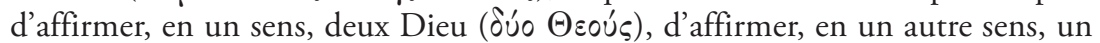

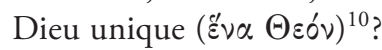

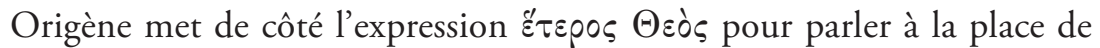
«deux dieux» et d'un «unique Dieu». Dans le passage biblique évoqué, le titre "premier-né de toute la création» vient immédiatement après «l'image de Dieu invisible» et est suivi par d'autres affirmations du rang divin du Fils de Dieu. Ce langage visiblement est plus compréhensible pour son interlocuteur qui exprime son approbation - mais le consensus est encore loin. Héraclide propose un argument décisif, à savoir la confession de foi en un «Dieu unique». Il le décrit comme «un Dieu qui est le tout-puissant, Dieu qui n'a pas de commencement et n'aura pas de fin, qui contient tout et n'est contenu par rien» et il n'est pas évident qu'il reconnaisse le même rang à celui qu'il appelle

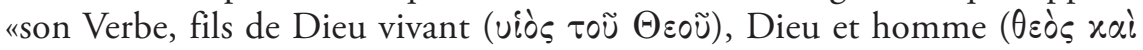
$\left.\alpha^{\prime} \nu \theta \rho \omega \pi \circ \varsigma\right)$, par qui toutes les choses se sont réalisées, Dieu selon l'Esprit et homme en tant qu'il est né de Marie» ${ }^{11}$.

Formulée ainsi, la doctrine confessée par Héraclide paraît, à première vue, irréprochable. Il parle de «Dieu unique» et de "Fils de Dieu» qui est «Dieu et homme». Il appelle "Dieu» l'un et l'autre, en réservant plutôt ó $\theta$ sò s au premier et $\theta$ sò s au second, mais cette nuance n'est pas exploitée dans l'échange qui suit. Cependant, dans la confession d'Héraclide il y a, pour ainsi dire, un angle mort, à savoir l'absence d'articulation entre les deux énoncés. Cela mène à un paradoxe qu'Origène ne manque pas de mettre en évidence:

Origène dit: (...) Le Père est Dieu?

Héraclide dit: Assurément.

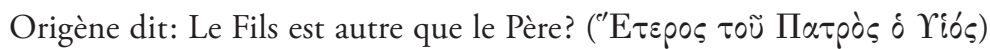

Héraclide dit: Sans doute! Comment être fils en étant en même temps père?

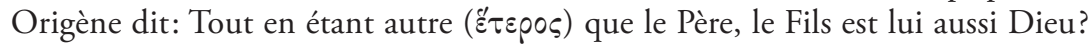

Héraclide dit: Il est lui aussi Dieu

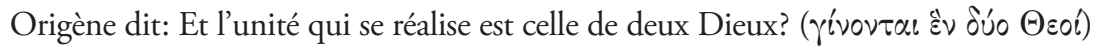

Héraclide dit: Oui

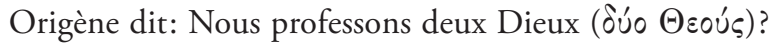

Héraclide dit: Oui. La puissance est une ${ }^{12}$.

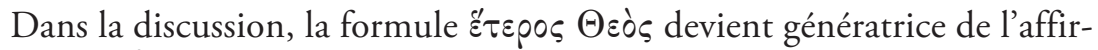

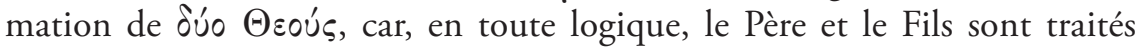
comme deux individus de la même espèce. Ce moment de l'entretien est particulièrement important pour la réflexion sur le dualisme, car il montre

10. Entretien 2, 3-6, éd. cit., p. 54-57.

11. Entretien 2, 7-13, éd. cit., p. 56-57.

12. Entretien 1,16 - 2, 27, éd. cit., p. 56-57. 
une difficulté inhérente à la doctrine chrétienne - et le débat entre les chrétiens et ceux qu'ils appellent Gnostiques s'articule autour de cette difficulté là.

Évidemment, l'échange entre Origène et Héraclide ne peut pas s'arrêter là: l'auditoire a besoin de comprendre pourquoi on lui annonce "deux Dieux", comment l'un est हैं $\varepsilon \rho \circ \varsigma$ par rapport à l'autre et, surtout, comment on peut désormais préserver l'unité de Dieu. Origène doit donc apporter un remède selon ses propres dires - au malaise général et expliquer sa position. Sans renoncer à l'idée de "deux dieux», il rassure son auditoire quant à l'unité de Dieu et donne presque aussitôt la solution:

[...] il faut montrer sous quel rapport ( $(\alpha \tau \tau \dot{\alpha} \tau \hat{\imath})$ ils sont deux, et sous quel rapport $\left(x \alpha \tau \dot{\alpha} \tau \tau^{\prime}\right)$ les deux sont un Dieu unique ${ }^{13}$.

Origène signale ici qu'une différence est nécessairement relative et que deux - ou plusieurs choses - peuvent former un «un", tout en restant

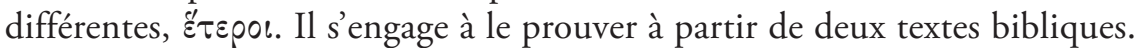
Le premier concerne le couple humain, où l'homme est la femme sont dits différents l'un de l'autre, mais ayant une chair en commun ${ }^{14}$ :

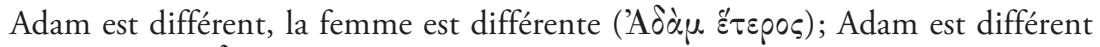

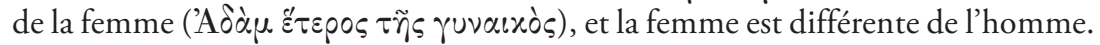
Or il est dit d'emblée dans la Genèse, que les deux sont «un»: car les deux seront une seule chair $\left(\sigma \alpha ́ p x \alpha \mu \alpha^{\prime} \alpha \nu\right)$ [...] Mais remarque bien que dans le cas d'Adam et Ève il n'est pas dit qu'ils seraient à eux deux un seul esprit, ni qu'ils seront à eux deux une seule âme, mais [...] une seule chair ${ }^{15}$.

Origène cite en exemple le couple biblique pour illustrer le passage de "Adam est différent» à "Adam est différent de la femme» et indiquer que cette différence est affirmée à propos des corps des êtres humains. L'exemple a pour but de montrer l'importance de ce par rapport à quoi les deux êtres sont différents et Origène tient à souligner qu'il ne s'agit pas d'être un esprit ( $\pi \nu \varepsilon u ̈ \mu \alpha$ है้ $)$ ni une seule âme ( $\left.\psi \cup \chi \hat{\eta} \nu \mu \dot{i}^{\prime} \alpha \nu\right)$, ce qui est très significatif pour la suite du discours.

Le second exemple biblique permet de mieux exposer l'importance du rapport, car la différence et l'unité interviennent entre le juste et le Christ au verset 1 Cor 6, 17:

Par ailleurs, le juste, tout en étant autre ( $\left.\varepsilon^{\prime} \tau \varepsilon \rho \rho \varsigma\right)$ que le Christ, est dit par l'apôtre

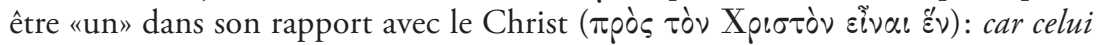
qui s'attache au Seigneur est un seul esprit avec lui. N'est-il pas vrai pourtant que

13. Entretien 2, 30-31, éd. cit., p. 58-59.

14. Cf. Gen 2, 24: «Les deux seront une seule chair»; Matt 19, 6: «... ne sont plus deux, mais une seule chair».

15. Entretien, 3, 5-12, éd. cit., p. 58-59. 
l'un est d'une essence (oủ $\sigma i \alpha)$ moins parfaite ou dégradée et inférieure, tandis que le Christ est d'une essence plus divine, plus glorieuse et [plus] bienheureuse ${ }^{16}$ ?

La différence dans le rapport entre le juste et le Christ concerne les essences, dont une est «moins parfaite» et l'autre "plus glorieuse». Cette manière de parler n'est pas très précise, car il peut s'agir de l'excès et du défaut d'une essence commune, plutôt que de deux essences différentes. En effet, Origène ne parle pas explicitement d'une substance humaine et d'une autre divine, probablement pour ne pas avoir à les opposer l'une à l'autre. Le théologien alexandrin se tient à la lettre de la Bible et évacue toute réflexion sur la substance. Ainsi il présente la différence et l'unité entre le juste et le Christ comme l'inégalité voire la différence par rapport à la substance (ou aux substances). Malgré la différence constatée, le juste et le Christ demeurent «un seul esprit» ${ }^{17}$.

Dans la suite de son discours, Origène prétend que les deux exemples cités sont semblables - ce qui n'est pas tout à fait exact - et passe à leur application au cas de «deux Dieux», qui sont à la fois différents et ne faisant qu'un:

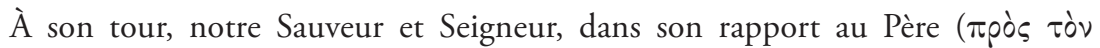
$\Pi \alpha \tau \hat{e}(\alpha)$ et Dieu de l'univers, est non pas une seule chair, non pas un seul esprit, mais - ce qui est supérieur à la chair et à l'esprit - un seul Dieu.

Il convenait alors, dans le cas d'êtres humains attachés l'un à l'autre, d'employer le mot «chair»; dans le cas de l'homme juste attaché au Christ, d'employer le mot «esprit»; et dans le cas du Christ uni au Père d'employer non le mot «chair», non le mot «esprit», mais un mot plus prestigieux que ceux-là, le mot «dieu» ${ }^{18}$.

À notre avis, cette conclusion, ainsi que le discours qui la précède, a besoin d'être lue à deux niveaux. Le premier est catéchétique: à l'aide des exemples bien orchestrés, Origène développe une argumentation en faveur de la divinité commune au Père et au Fils, convaincante pour tout chrétien. Mais il y a aussi un deuxième niveau de lecture possible, car on constate que les deux exemples bibliques illustrent des catégories philosophiques du différent et du relatif. Il est vrai aussi que ces deux catégories aident à expliquer l'expression

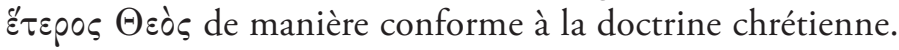

Pour terminer son analyse, Origène évoque un exemple surprenant, celui de l'«autre Dieu» au sens de dieu d'un autre peuple ou des dieux des religions

16. Entretien, 3, 13-17, éd. cit., p. 58-61.

17. Selon Origène l'esprit - et non la partie supérieure de l'âme - est un lieu d'union entre l'homme et Dieu. On peut renvoyer à l'étude de J. Dupuis, qui montre le pneuma comme un élément commun à Dieu et l'homme; cf. J. Dupuis, L'Esprit de l'homme. Étude sur l'anthropologie religieuse d'Origène, Desclée de Brouwer, 1977.

18. Entretien 3, 20-28, éd. cit., p. 60-61. 
polythéistes. Il cite alors deux versets de l'Ancien Testament (Is 43, 10 et

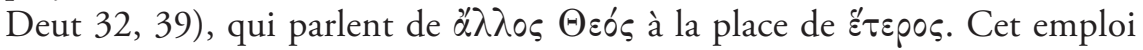
est sans rapport avec la différence entre le Père et Fils discutée avec Héraclide et on peut se demander pourquoi Origène a cru bon de terminer son discours de cette manière, si ce n'est pour montrer l'équivalence des adjectifs $\ddot{\alpha} \lambda \lambda \circ \varsigma_{5}$

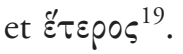

\section{Dualisme et vocabulaire d'Origène}

Une lecture attentive de l'échange avec Héraclide permet de constater l'importance du choix de vocabulaire. Il est clair que le terme हैं $\varepsilon \rho \circ \varsigma$, et

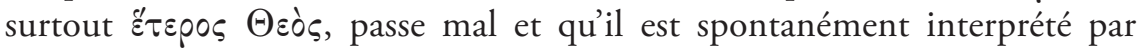
l'assemblée dans le sens «deux Dieux». Pire encore, cette expression semble éveiller les démons du paganisme et de l'hérésie.

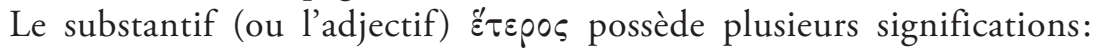
«autre», «distinct», «différent» ou «l'un des deux». Sa signification de base est «l'autre» ou «l'un des deux». Parfois il se traduit aussi par «second». Le terme

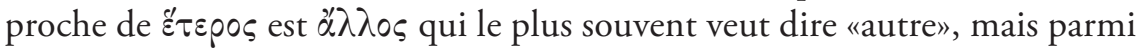
des nombreux éléments. On peut donc dire que हैं $\varepsilon \rho \circ \varsigma$ se prête mieux à des lectures dualistes, bien que ceci ne soit pas une règle, car les deux termes sont

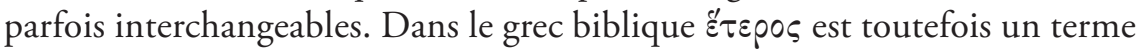

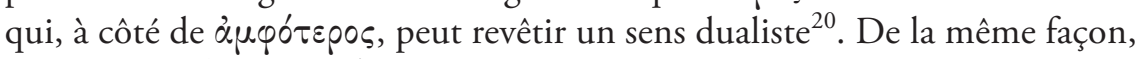

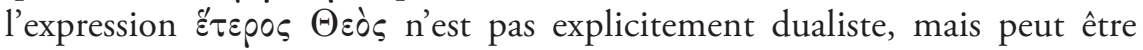

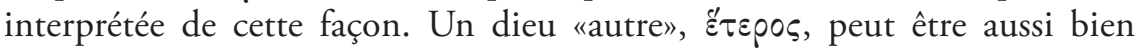
différent qu'opposé. Ainsi l'utilisation de ce terme fait éclater un débat sur la différence et l'opposition, comme des approches possibles du dualisme.

Il existe en grec un vocabulaire philosophique riche et précis pour exprimer la différence, dont témoignent surtout les Catégories d'Aristote. Dans la mesure où certains éléments de la dialectique aristotélicienne avaient passé dans la philosophie courante, ils peuvent se retrouver chez Origène ${ }^{21}$. À notre

19. Cf. Entretien 4, 9-15. Cette équivalence à déjà été introduite par Héraclide au début de l'échange; $c f .2,1-2$.

20. Cf. F. Blass, A. Debrunner, Grammatik des neutestamentlischen Griechisch, Göttingen, 1976; J.H. Moulton, G. Miligan, The Vocabulary of the Greek Testament, London, 1914-1929.

21. Le rapport d'Origène à Aristote est complexe. Il est vrai qu'Origène - comme les auteurs chrétiens en général - critique chez les Péripatéticiens la négation de l'immortalité de l'âme et de la providence, ainsi que le manque d'intérêt pour la prière, les miracles et les visions. Cependant, les travaux de A.-J. Festugière (L'idéal religieux des Grecs et l'évangile, Paris, 1932) et de D. Runia («Festugière Revisited: Aristotle and the Greek 
avis, la classification des opposés faite par Aristote avait inspiré la lecture des exemples bibliques dans l'Entretien. Dans le Catégories et dans le livre $\Delta$ de la Métaphysique il y a plusieurs passages qui traitent des opposés et des relatifs. Tout d'abord, les opposés ( $\alpha \nu \tau \iota x \varepsilon i ́ \mu \varepsilon \nu \alpha)$ sont divisés en quatre classes:

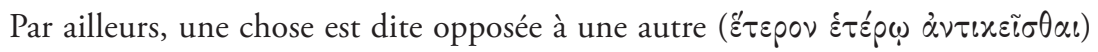
de quatre façons: soit à la façon des relatifs ( $\tau \dot{\alpha} \pi \rho o ́ \varsigma ~ \tau \iota)$, soit à la façon des

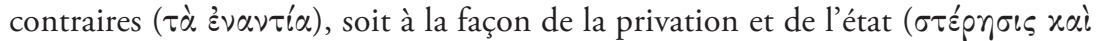

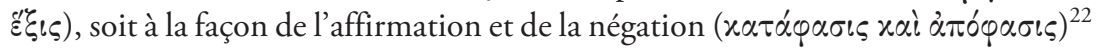
(11b 17-19).

Dans la Métaphysique $\Delta$, la même classification des opposés se prolonge dans une réflexion sur «autre»:

Autre selon l'espèce ( $\left.\left.\varepsilon^{\prime} \tau \varepsilon \rho \alpha \delta \varepsilon^{\prime} \tau \tilde{\varphi}\right) \varepsilon \iota^{\prime} \delta \varepsilon \iota\right)$ s'applique aux êtres qui, étant du même

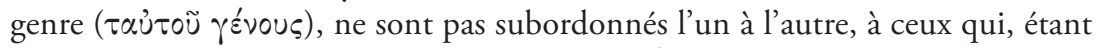

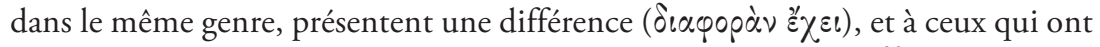

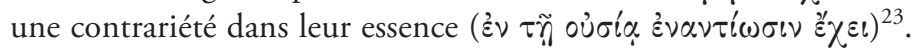

Ces deux fragments éclairent l'argumentation origénienne ainsi que son choix des versets bibliques. L'exemple du juste et du Christ semble s'inspirer de l'idée de contrariété dans la substance, l'exemple du couple humain ressemble au cas des êtres du même genre présentant une différence. Penser le Père et le Fils comme ع́ $\tau \varepsilon \rho \circ$, l'un par rapport à l'autre, va avec l'idée de l'absence de la subordination dans leur rapport. L'audace avec laquelle Origène

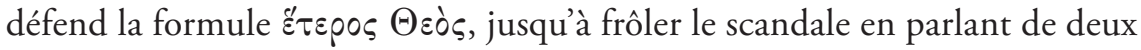
Dieux, vient sans doute du fait qu'il fait bien la distinction entre «opposé»

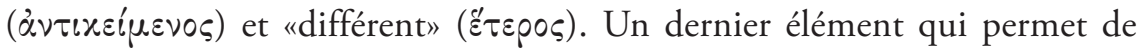
parler de référence aux catégories aristotéliciennes, est le relatif $-\chi \alpha \tau \dot{\alpha} \tau \iota$ et

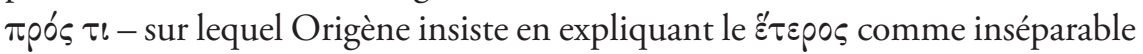
d'une relation entre deux êtres. Or, certains contraires peuvent être à la fois des relatifs ou des corrélatifs ${ }^{24}$. Le discours d'Origène montre une connaissance

Patres», Vigiliae christianae, n 43, 1989, p. 1-34) montrent une importante présence des références à Aristote dans les écrits patristiques. Sur le rapport d'Origène à Aristote: M.J. Edwards, Origen Against Plato, Ashgate, 2002.

22. Cat. 11b 17-19, trad. R. Bodéüs, Paris, Les Belles Lettres, 2001, p. 53; cf. Mét. $\Delta, 1018 \mathrm{a} 25-37$.

23. Mét. $\Delta, 1018 \mathrm{a} 38$ - 1018b8, trad. J. Tricot, Paris, Vrin, 1991, p. 187 . Voir aussi Mét. $\Delta, 1018 \mathrm{a}-\mathrm{b}$ : «Autre se dit des êtres qui ont pluralité d'espèce, ou de matière, ou de définition de leur substance, et, d'une manière générale, l'autre présente des significations opposées à celles du même».

24. Cf. Cat. 6b 30-31; Mét. $\Delta, 1020$ b25 - 1021 b10. 
vague des catégories aristotéliciennes qui sont traitées comme des outils conceptuels, déconnectés de la doctrine: par exemple, pour Aristote, l'homme

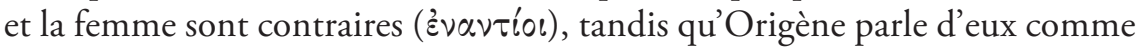
différents (हैं $\varepsilon \rho \circ \iota)$.

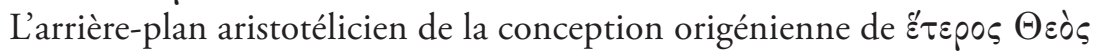
explique sa méthode d'argumentation, mais notre véritable problème concerne les raisons théologiques qui ont poussé Origène à chercher un sens, pour

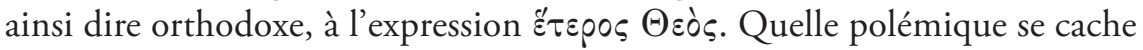
derrière l'échange avec Héraclide? Le texte ne fournit pas de réponses claires. À la fin du discours, Origène parle ainsi de ses adversaires:

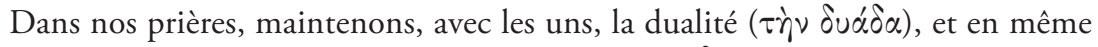

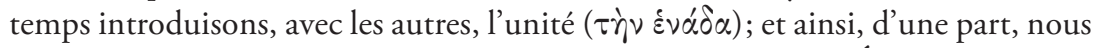
ne tombons pas dans l'opinion de ceux qui se sont séparés de l'Église pour verser dans l'illusion de la monarchie, supprimant le Fils et le retirant du Père; et, d'autre part, nous ne tombons pas dans une autre doctrine impie, celle qui nie la divinité du Christ ${ }^{25}$.

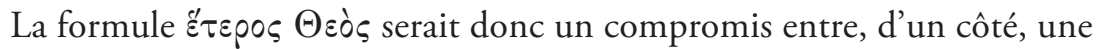
forme du dualisme qui voit le Dieu unique comme opposé au Christ dont il nie la divinité; de l'autre côté, une forme du monarchianisme qui tend à supprimer la différence entre les deux. On retient d'abord les informations sur les Monarchianistes «qui se sont séparés de l'Église» et sur un groupe professant «une autre doctrine impie», mais opposée à la précédente. Depuis la fin du $\mathrm{II}^{\mathrm{e}}$ s. et au $\mathrm{III}^{\mathrm{e}}$ s., les différentes formes du monarchianisme sont connues en Asie Mineure, à Antioche, à Rome et en Afrique du Nord. Les principaux représentants de cette doctrine (Théodote de Byzance, Noët de Smyrne, Sabellius) furent excommuniés, malgré leur ambition de combattre

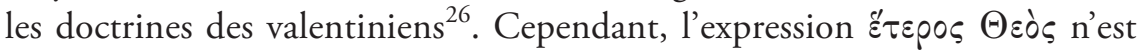
pas typiquement valentinienne, mais plutôt marcionite. Selon Marcion, le Dieu de l'Ancien Testament est un Créateur démiurgique, tandis que le Dieu suprême est "autre», "étranger», «inconnu», "séparé»" 27 Marcion donnerait

25. Entretien 4, 2-9, éd. cit., p. 60-63.

26. Cf. R.M. Hübner, Der paradox Eine. Antignostischer Monarchianismus um zweiten Jahrhundert, Leiden/Boston/Köln, Bril, coll. «Supplements to Vigiliae Christianae», $\mathrm{n}^{\circ} 50,1999$.

27. Cet aspect a été souvent mis en évidence par les différentes doctrines gnostiques; cf. A.-J. Festugière, La Révélation de Hermès Trismégiste. IV- Le Dieu inconnu et la gnose, Paris, 1954; H. Jonas, La religion gnostique. Le message du dieu étranger et les débuts du christianisme, Paris, Flammarion, 1992; S. Pétrement, Le Dieu séparé. Les origines du gnosticisme, Paris, Cerf, 1984, p. 50-56; A. von Harnack, Marcion, l'évangile du Dieu étranger, Paris, Cerf, 2005, p. 140-143. 


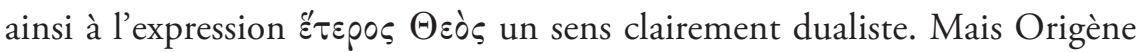
ne nous dit pas qu'il s'agit de Marcion.

Il est très significatif que la manière de présenter les positions extrêmes ne soit pas symétrique, car le terme "monarchianisme schismatique» comporte davantage de précision qu'une "doctrine impie», dont les représentants sont susceptibles de faire toujours partie de la communauté chrétienne. En outre, Origène s'adresse à ceux qui - comme l'évêque Héraclide - adhèrent partiellement à l'une des deux doctrines incriminées, ne serait-ce qu'au niveau des formulations. Pour illustrer l'attitude d'Origène, on pourrait évoquer le recueil hérésiologique intitulé Elenchos (connu aussi comme Philosophoumena) ou Réfutation de toutes les hérésies ${ }^{28}$. Le lecteur moderne constate que la liste des doctrines réfutées est particulièrement longue et variée: les philosophes, les brahmans, les magiciens et les druides, les écoles gnostiques, quelques auteurs chrétiens (même un évêque de Rome), les Juifs - ils sont tous logés à la même enseigne. On peut alors admettre que pour Origène une doctrine «impie» et condamnable ne signifie pas automatiquement une doctrine schismatique, ce qui correspond d'ailleurs au tableau particulièrement complexe des rapports entre les gnostiques et les communautés chrétiennes. Ainsi, le

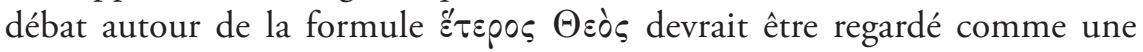
recherche de compromis entre les énoncées fondamentaux de la foi chrétienne et les positions radicales représentées par les Monarchianistes et, probablement, par les Marcionites ${ }^{29}$.

28. La tradition manuscrite attribue l'Elenchos à Origène et depuis 1853 cette œuvre a été attribuée à Hippolyte de Rome. La question de l'auteur de cet ouvrage a été soulevée en 1947 par des publications de P. Nautin qui partageait le corpus hippolytéen entre deux auteurs - Hippolyte et Josipe -, et attribuait au second la Réfutation avec deux autres écrits. Sa proposition a d'abord été rejetée (J. Frickel, M. Marcovich, M. Richard, etc.), avant d'être reprise et reformulée par V. Loi et surtout par M. Simonetti qui propose un «Hippolyte oriental», différent du romain. L'hypothèse de Simonetti a donné lieu à de nombreux rebondissements dans la recherche ( $c f$. G. Aragione, «Bibliographie sur l'Elenchos et la question de l'auteur (1940-2010)», dans G. Aragione, E. Norelli (dir.), Des évêques, des écoles et des hérétiques. Actes du colloque international sur la "Réfutation de toutes les hérésies» Genève, 13-14 juin 2008, éditions du Zèbre, 2011, p. 315-323). Actuellement, on parle plutôt de «l'auteur de l'Elenchos» ou de «Hippolyte II» (cf. C. Scholten, «Hippolytus», $R A C$ 15, col. 492-551), sans exclure la possibilité d'avoir des écrivains homonymes, provenant du même milieu romain.

29. Dans l'analyse de ce débat, M. Simonetti attribue les positions contraires aux Monarchanistes et aux Docètes, sans expliquer cette identification. Certes, la négation de la divinité du Christ peut coïncider avec le docétisme, mais elle ne lui est pas exclusive; cf. M. Simonetti, Studi sulla cristologia del II e III secolo, op. cit., p. 139-143. 


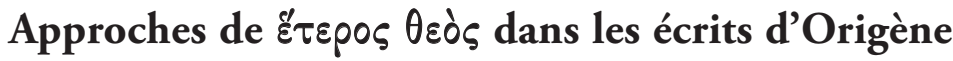

L'entretien avec Héraclide n'est ni la seule, ni la première occasion pour

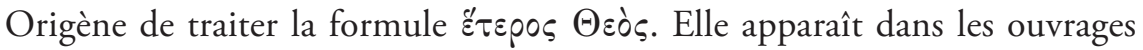
consacrés entièrement ou partiellement à la polémique anti-gnostique (Commentaire sur l'Évangile selon Jean, Sur les principes, Contre Celse), principalement en tant que formule utilisée par les adversaires d'Origène. Le meilleur exemple de cette attitude vient du Commentaire sur Jean (rédigé dans les années 231-238), qui est une réponse au commentaire du même évangile par un Gnostique valentinien, Héracléon ${ }^{30}$, dédicacée à un ami converti du valentinisme. Néanmoins, le commentaire contient des arguments dirigés non seulement contre les valentiniens et leur multitude de $\lambda$ ó $\gamma o \iota$, mais aussi contre les Marcionites professant un «autre Dieu» ${ }^{31}$. Dans le traité Contre Celse (env. 248-249), Origène dénonce ouvertement les idées marcionites attribuées par Celse à la majorité des chrétiens ${ }^{32}$, tandis que dans Sur les principes (rédigé vers 229-230), la critique anti-gnostique marque la structure et la thématique de l'ouvrage, mais elle est moins ouvertement déclarée ${ }^{33}$.

Dans ce contexte polémique particulièrement complexe, Origène est

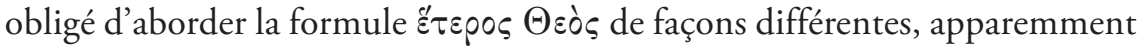

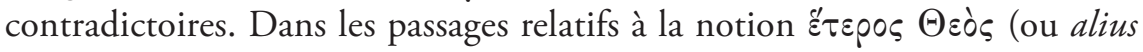
deus) nous trouvons surtout des critiques explicites. On peut les diviser en deux groupes, selon le sens attribué à cette expression. En premier lieu vient une lecture nettement dualiste qui oppose un Dieu à l'autre. Il s'agit surtout d'une idée marcionite d'opposer le Dieu juste de l'Ancien Testament au Dieu bon annoncé par Jésus-Christ, mais qui avait également des conséquences christologiques. Cette réflexion se prolonge dans un cas particulier Satan (et l'Antéchrist) décrit comme évavtíos, contraire. La deuxième interprétation concerne tous les êtres spirituels qui sont «autres dieux». On commence par des êtres participant à la nature divine (tels les anges), mais qui peuvent également devenir des démons. Dans ce contexte apparaît aussi la notion de "faux dieux».

30. Le Commentaire d'Origène est notre seule source d'information au sujet d'Héracléon qui fut probablement un valentinien; $c f$. Y. Janssens, "Héracléon. Commentaire sur l'Évangile selon saint Jean", Le Muséon, n 72, 1959, p. 101-151; p. 277-299.

31. Les principaux passages anti-marcionites: ComJn V, 7. 8; II, 28.

32. Cf. CCelse VI,74.

33. Sur le caractère anti-gnostique de Sur les principes: A. Le Boulluec, «La place de la polémique antignostique dans le Peri Archon», dans H. Crouzel, G. Lomiento, J. Rius-Camps (dir.), Origeniana. Premier colloque op. cit., p. 49-61; voir dans le même volume les articles de M. Alexandre et J. Rius-Camps. 
Commençons par la possibilité d'appeler le Christ «autre Dieu». Il semble qu'Origène ne le fasse nulle part, en dehors de l'Entretien avec Héraclide. Dans le traité Sur les principes (Peri Archon), cette formule est une réponse au modalisme d'origine gnostique (ou autre) ${ }^{34}$. Déjà dans la préface, Origène annonce clairement les vérités de la foi, parmi lesquelles figure l'affirmation que Jésus-Christ «s'est incarné, alors qu'il était Dieu, et devenu homme, il est resté ce qu'il était, Dieu ${ }^{35}$ ". En revanche, dans la section consacrée au Fils, il le présente au travers d'expressions scripturaires, telles que Sagesse de Dieu, Puissance, Parole, Vérité, Vie, Image, Rayonnement de la gloire, Figure, Souffle, etc. Il ne s'agit pas simplement des noms ou attributs du Christ,

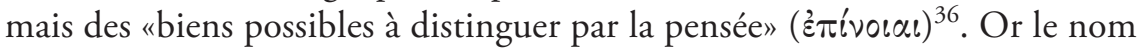
«Dieu» ne figure pas sur la liste, ce qui est cohérent avec les textes bibliques. La divinité et l'unité du Christ-Logos sont aussi le sujet du premier livre du Commentaire sur Jean. À chaque fois, en passant par les nombreux é $\pi i^{\prime} v o \iota \alpha \iota$ du Christ, Origène veille à ce que chacune soit présentée aussi comme relative à Dieu le Père, ce qui empêche de transformer le Christ en un dieu «autre», au sens de «séparé du Père».

Le point de départ des différentes lectures du terme «autre Dieu» est la polémique anti-marcionite et le refus de cette formule au nom de l'unité de l'Ancien et du Nouveau Testament. Ce point correspond à la méthode d'Origène de prendre les Écritures pour norme et référence dans son discours hérésiologique ${ }^{37}$. En se référant aux Marcionites, Origène rappelle dans un autre passage du Commentaire sur Jean que leur doctrine attribue deux Testaments à deux dieux différents:

34. Cette question occupe le premier livre de PArch I, 2, 2; I, 2, 3; I, 2, 4; I, 2, 10 ; I, 2, 13. Sur la critique du modalisme dans cet ouvrage, $c f$. Le Boulluec, «La place de la polémique antignostique dans le Peri Archon», dans Origeniana. Premier colloque international des études origéniennes (Montserrat, 18-21 septembre 1973), H. Crouzel, G. Lomiento, J. Rius-Camps (éds), coll. Quaderni di «Vetera Christianorum», n 12, 1975, p. 54. La même question occupe aussi le premier livre du Commentaire sur Jean.

35. PArch, pref. 4; trad. H. Crouzel, M. Simonetti, SC 252, Paris, 1978, p. 80-81.

36. «Le Sauveur est, comme nous l'avons vu, beaucoup de biens que la pensée distingue; il ne faut donc pas s'étonner que certains de ces biens soient premiers, d'autres seconds ou troisièmes. Parlant du Logos, Jean a affirmé: "Ce qui fut fait en lui était vie". La vie fut donc faite dans le Logos, mais le Logos ne diffère par du Christ, le Dieu Logos qui est auprès du Père et par qui toutes choses ont été créées, et la vie ne diffère pas du Fils de Dieu qui dit: "C'est moi le chemin, la vérité et la vie "”. ComJn I, 19, 112; SC 120, p. 119; voir aussi: CommJn I, 28, 200; X, 5, 21; XIX, 6, 38.

37. Cf. M. Harl, «Pointes anti-gnostiques d'Origène; le questionnement impie des écritures», dans R. Van Den Broek, M .J. Vermaseren (dir.), Studies in Gnosticism and Hellenistic Religions presented to G. Quispel, Leiden, 1981, p. 205-217; A. Le Boulluec, "L'écriture comme norme hérésiologique dans les controverses des II $^{\mathrm{e}}$ et III $^{\mathrm{e}}$ siècles (domaine grec)», dans Stimuli. Festschrift für E. Dassmann, coll. "Jahrbuch für Antike und Christentum. Ergänzungsband», n 23, 1966, p. 66-76. 
Je me demande donc comment font les hérétiques pour attribuer les deux testaments à des dieux différents (oi $\delta$ vii $\theta \varepsilon o i c s)$, car ce texte les convainc encore davantage de l'erreur [litt. pas moins]. En effet, comment Jean peut-il être le début de l'Évangile,

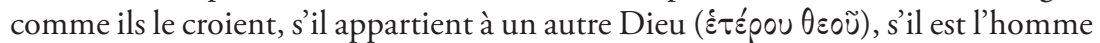
du démiurge et ignore, selon leurs imaginations, la nouvelle divinité ${ }^{38}$ ?

Plus loin, il parle des conséquences de cette doctrine qui se manifestent également dans la christologie:

Parmi les hérétiques, certains, prétendant croire au Christ, mais forgeant un second

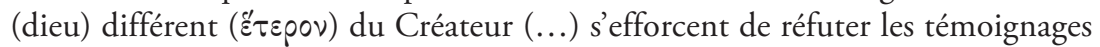
rendus au Christ par les prophètes et affirment que le Fils de Dieu n'a pas besoin de témoins. ${ }^{39}$

Par la suite, Origène reconnaît que sans le témoignage des prophètes, le Christ peut être cru grâce à ses paroles et ses miracles, mais qu'il y a aussi d'autres conséquences, autrement plus graves. En introduisant une divinité «autre» à côté du Dieu unique, les Marcionites opèrent le même dédoublement pour le Fils de Dieu: l' «autre Dieu» doit avoir un «autre Fils». Sur les conséquences de l'expression Fils de Dieu nous renseigne un fragment de l'écrit de Celse rapporté par Origène comme exemple de mélange condamnable entre la doctrine marcionite et le christianisme «orthodoxe»:

Alors, de nouveau il badine et raille à son habitude, introduisant deux fils de dieu: l'un, fils du démiurge, l'autre de Dieu, d'après Marcion. Il décrit leurs combats singuliers et les dit «combats de cailles», comme l'étaient ceux des dieux leurs pères: rendus par la vieillesse inutiles et radoteurs, incapables d'animosité réciproque, ils laissent combattre leurs enfants ${ }^{40}$.

Origène dénonce, une fois de plus, l'inspiration marcionite des propos de Celse. Ayant introduit deux Dieux opposés, il est impossible que le Christ, annonçant le Père bon et miséricordieux, puisse être fils du mauvais Créateur de l'Ancien Testament - il faut qu'il y en ait un autre, celui dont parlent les prophètes juifs. Toutefois, cette idée a dû pleinement convenir à Celse qui ne voulait pas identifier le Logos de Dieu avec «un homme ignominieusement battu des verges et conduit au supplice» ${ }^{41}$.

38. ComJoh I, 13, 82; Blanc, SC 120, p. 98-99.

39. ComJoh II, 199; Blanc, SC 120, p. 346-349. La même mention se trouve en VI, 31 - «certains ont été jusqu’à forger un second dieu plus grand (que le premier)».

40. CCelse VI, 74, 11-17; Borret, SC 147, p. 364-367. Voir aussi CCelse V, 54; VI, 52-53.

41. CCelse II, 31; Borret, SC 132, p. 362-363. Le développement de idée se trouve aussi en II 28-30. 
En fait, la place accordée à Jésus-Christ et son titre de Fils de Dieu rendent le monothéisme des Chrétiens pour le moins suspect aux yeux de Celse. Origène cite son opinion sur ce sujet:

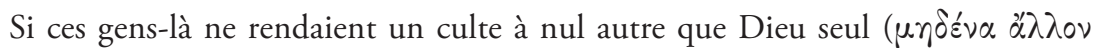

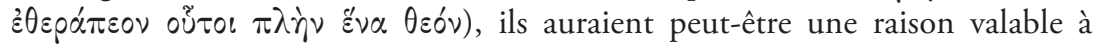
opposer aux autres. Mais non, ils rendent un culte excessif à celui qui vient d'apparaître, et pourtant ils ne croient point offenser Dieu en rendant aussi un culte à son ministre ${ }^{42}$.

Cette critique rappelle vaguement le débat résumé dans l'Entretien avec Héraclide, car on peut constater que Celse et l'évêque Héraclide partagent la même difficulté concernant la divinité du Christ dans son rapport à l'unicité de Dieu. Mais la réponse d'Origène n'est pas la même dans les deux cas. Contre Celse, il commence par évoquer rapidement des versets johanniques semblables à «Le Père et moi sommes un» (Jn 10, 30; 17, 21-22; 14, 10-11; 17,21 - avec quelques mises en garde contre «ceux qui nient l'existence de deux hypostases». Ses explications portent principalement sur la question du culte communément rendu au Père et au Fils. Au cours de ce discours, Origène précise qu'ils sont "deux réalités par hypostase, mais une seule par

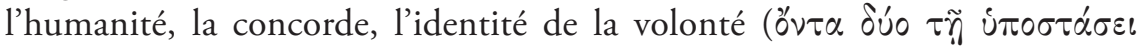

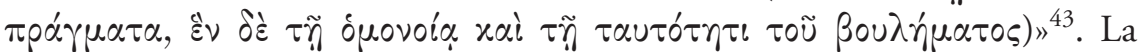
logique adaptée par Origène dans l'une et l'autre réponses montre très bien la différence dans les approches de l'expression «autre dieu». Tandis que l'évêque Héraclide fait probablement partie de ceux qui ont des difficultés avec les deux hypostases divines, Celse brandit l'accusation de polythéisme caché dans le culte rendu au Fils de Dieu. S’il y a un point commun entre les objections d'Héraclide et de Celse, la ressemblance s'arrête ici. Sans surprise, Celse organise son argumentation autrement. En accusant les chrétiens de compromettre leur monothéisme dans l'imitation maladroite de la religion traditionnelle, quelques pages plus haut, il entend cet «autre dieu» comme un adversaire du Dieu unique.

Après quoi, il déclare: «En vérité, celui qui affirme qu’un seul être a été appelé Seigneur, en parlant de Dieu, commet une impiété: il divise le Royaume de Dieu et $\mathrm{y}$ introduit la révolte, comme s'il y avait une faction et un autre dieu son

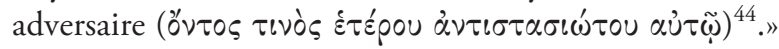

42. CCelse VIII, 12; Borret, SC 150, p. 198-199. Voir aussi CCelse I, 26 et VI, 10.

43. CCelse VIII, 12; Borret, SC 150, p. 200-201; cf. M. Fédou, Christianisme et religions païennes dans le Contre Celse d'Origène, Paris, Beauchesne, coll. "Théologie historique», n 81, 1988, p. 282-288.

44. CCelse VIII, 11; trad. Borret, SC 150, p. 196-197. 
Origène répond qu'il ne s'agit pas des dieux mais des mauvais démons, dont aucun ne mérite le titre «autre dieu». Voici donc une autre raison de réfuter farouchement ce titre au Christ. Il conclut ainsi:

Il n'y a donc pas chez Dieu de factions ni un autre dieu, son adversaire; et cela en dépit de ceux qui, à l'instar des Géants et des Titans, veulent par leur perversité batailler contre Dieu avec Celse et les autres qui ont déclaré la guerre à Dieu qui a établi par tant de moyens la vérité sur Jésus ${ }^{45}$.

Lidée de l'«autre dieu» exposée ici par Celse paraît très complexe. En partant des exemples des guerres des dieux racontées par Homère et ses commentateurs, il n'a pas de doute quant à l'introduction d'un dieu «autre» qui conduit à une division dans le royaume divin et définitivement produit des batailles entre les immortels. Il interprète donc «autre» comme potentiellement «opposé». Toutefois, le projet de Celse n'est pas celui d'opposer Jésus à Dieu, mais plutôt de présenter le culte qui lui est rendu par les chrétiens comme un blasphème contre le Dieu unique - la réponse donnée par Origène va précisément dans ce sens.

Néanmoins, Celse n'abandonne pas complètement ses critiques du monothéisme chrétien. Dans le livre VI, Origène cite un très long fragment, dans lequel Celse explique que les chrétiens n'ont rien compris à ce thème homérique lorsqu'ils parlent de Satan en faisant de lui un adversaire de Dieu qui a même châtié le Fils de Dieu. Celse rappelle que, pour les païens, la guerre des dieux est une allégorie de la création mais, introduite telle quelle dans les écrits des chrétiens, elle compromet leur idée d'un Dieu unique ${ }^{46}$, car ils font de Satan un "opposé» de Dieu, donc une version radicale de l'«autre dieu». Origène consacre plusieurs pages à expliquer qui est Satan et qui est l'Antéchrist. Il insiste pour que ces deux derniers ne soient pas compris comme des divinités

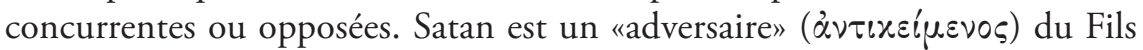
de Dieu, un être qui est tombé de son état bienheureux. Origène rejette tout aussi vigoureusement une vision dualiste du Christ et de l'Antéchrist:

Est-il donc absurde qu'il y ait dans l'humanité pour ainsi dire deux extrêmes,

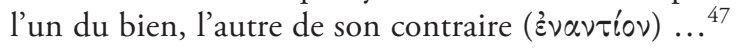

45. CCelse VIII, 11; trad. Borret, SC 150, p. 198-199. Sur les Titans et Géants adversaires de Dieu: comme démons terrestres (IV, 23), comme Ophites et partisans de Celse (VI 28). Voir aussi: CCelse VII, 66.

46. Cf. CCelse VI, 42.

47. CCelse VI 45; Borret, SC 147, p. 290-291. Une proposition bien différente de celle d'Origène est exprimée par Hippolyte de Rome dans l'écrit Sur l'Antéchrist. Il écrit

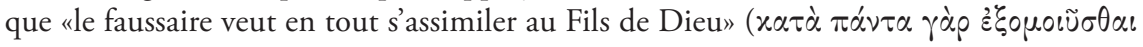


Devant une proposition aussi radicale, Origène évacue soigneusement le

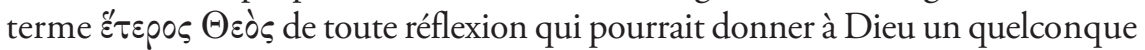
adversaire.

Le même raisonnement s'applique au débat sur la nature du «vrai dieu» qui oppose les divinités traditionnelles au Dieu des chrétiens. Or Celse, n'étant pas un inconditionnel de la mythologie, développe une argumentation fondée principalement sur la critique formulée par Héraclite qui porte sur la question de la nature du vrai dieu ${ }^{48}$. Selon Celse, il est donc stupide de prier les statues quand on ne connaît pas la vraie nature des dieux et des héros, à quoi Origène répond avec quelques arguments traditionnels contre le polythéisme:

Il est possible de connaître Dieu et son Fils unique, comme les êtres qui sont honorés par Dieu du titre dieu et participent à sa divinité, et qui sont différents

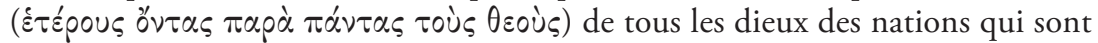
de leur vraie nature des démons; mais il n'est pas possible en vérité de connaître Dieu et de prier les statues ${ }^{49}$.

Mais son discours rebondit sur la question de «l'autre dieu»:

Nous refusons ainsi d'honorer les statues pour éviter ... de tomber dans l'opinion que les statues seraient d'autres dieux ( $\theta$ coù è érépouৎ $)^{50}$.

Pour commencer, Origène refuse toute comparaison entre les démons et Dieu avec son Fils unique. Les "démons» ne sont pas les «dieux», dit-il. Cependant, il doit tout de même reconnaître que les Écritures parlent des «autres dieux», des êtres qui participent à la divinité - mais qui toujours restent rigoureusement différents des divinités païennes de nature démoniaque. Le thème de l'adoration des images est certes secondaire par rapport à la question de l'unicité de Dieu, mais il éclaire la conception de l'«autre dieu» qui est en jeu. Même si la Bible parle des "autres dieux», cela n'est pas un argument pour affirmer la divinité de Jésus - en tout cas, il n'en est pas un pour Origène.

Dans un contexte moins directement polémique, à savoir dans un long passage de Sur les principes consacré aux «créatures raisonnables» et «puissances

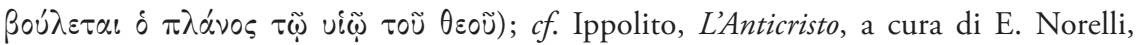
Firenze, Nardini Editore, 1987.

48. Le fragment d'Héraclite: «Et encore ces statues qu'ils prient comme si l'on bavardait avec des maisons. Ils ne savent rien de la vraie nature des dieux et des héros?» Frg B 5 (Diels - Kranz I); cf. CCelse VII, 62.

49. CCelse VII, 65, 21-25; Borret, SC 150, p. 166-167.

50. CCelse VII, 66; Borret, SC 150, p. 168-169. 
adverses» Origène propose une explication de la différence entre ces «dieux» et le Dieu unique ${ }^{51}$. Celle-ci consiste dans le caractère accidentel de la vertu: les puissances peuvent devenir des saints ou des démons. Origène dit qu'elles "peuvent recevoir l'état contraire» (possint recipere omnino contrarium), parce qu'elles possèdent le libre arbitre et parce que «le bien n'est pas en elles quelque chose de substantiel» (id est quia non substantiale sit in ipsis bonum). Ainsi celles qu'il appelle "puissances mauvaises et contraires» deviennent telles suite à un changement réalisé dans leur libre arbitre et pas par la décision du Créateur. Leur élévation ou leur déchéance arrive "par suite de leurs mérites et non par une prérogative due à leur création.» En ces termes, Origène non seulement refuse l'idée que Dieu ait pu faire les créatures bonnes les unes et mauvaises les autres, mais formule une série d'arguments qui articulent la différence entre elles et Dieu. Tout d'abord, Dieu étant par nature simple, sans composition (I, 5, 3), il ne peut pas subir de changements; puis, étant bon et saint de façon substantielle (substantialiter), il ne peut pas perdre cette qualité (I, 5, 5). Les conclusions ultérieures sont cependant anthropologiques:

Ces puissances contraires ont été autrefois immaculées et se sont trouvées au milieu de celles qui restent encore immaculées: cela montre que personne n'est immaculé de façon substantielle ou naturelle, ni souillé de façon substantielle. Il s'ensuit qu'il dépend de nous et de nos mouvements d'être saints et bienheureux, ou bien par paresse et négligence de nous écarter de la béatitude pour tomber dans la malice et dans la perdition $(\ldots)^{52}$.

Cette argumentation est très significative pour la question du dualisme. D'abord, elle vise à refuser la dénomination «dieu» à un être qui aurait réuni en lui toutes les caractéristiques contraires à celles du Dieu unique. Origène propose une solution paradoxale, une situation où l'«autre dieu", pour être «dieu» doit posséder sa perfection, sa bonté, sa sainteté de manière tout aussi substantielle, donc immuable. L' «autre dieu» ne peut exister en tant que contraire voire opposé, car il soumis aurait se devenir, en abandonnant ainsi son immuabilité. Ainsi il serait dieu de manière impropre, de la même manière que les puissances des cieux sont dites «dieux», et c'est à elles qu'il serait contraire. Par conséquent, ce raisonnement sert à expliquer l'origine du bien et du mal chez les êtres humains et à défendre leur libre arbitre.

Cet examen des écrits origéniens permet de conclure au caractère exceptionnel du discours tenu dans l'Entretien avec Héraclide. Confronté aux arguments de Celse, partiellement d'inspiration marcionite, il est habitué à entendre

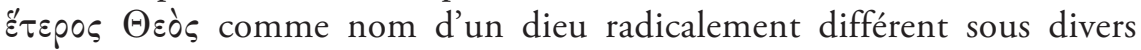

51. Un long passage dans Sur les principes I, 5-6 traite de «créatures raisonnables» et de "puissances contraires», avec des explications quant à leur caractère accidentel.

52. PArch I, 5, 5; Crouzel, Simonetti, SC 252, p. 192-195. 
aspects: tantôt comme Créateur de l'Ancien Testament, tantôt comme Adversaire susceptible de diviser le royaume. Dans ces deux cas, Origène s'oppose à une

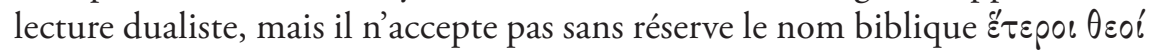
attribué aux êtres qui partagent la nature divine.

Dans le Commentaire sur Jean, il examine l'expression biblique «Dieu des dieux». Qui sont donc ces «dieux»? Origène explique cela par rapport à un verset de la lettre aux Colossiens où il est question de ces «autres» en tant que trônes, dominations, principautés et puissances $\left(c f\right.$. Col 1, 16) ${ }^{53}$. Et il conclut ainsi:

... il faut croire qu'il existe encore d'autres êtres doués de raison, différents ( $\left.\alpha^{\prime} \lambda \lambda \alpha\right)$ de ceux-ci et que nous n'avons pas coutume de nommer: l'Hébreu a donné à l'une de ces espèces ( $\gamma$ ร́vos) le nom de «Sabaï», d'où vient "Sabaoth», le maître

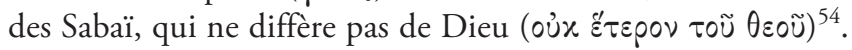

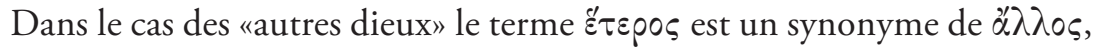
donc un autre parmi d'autres, un cas particulier de la même espèce. Il n'est pas étonnant qu'Origène n'entreprenne pas le même raisonnement dans l'Entretien avec Héraclide - il ne présente pas le Fils de Dieu comme l'un des "autres dieux», au risque de faire de lui l'un des anges. Cependant, ses prédécesseurs Justin Martyr, par exemple - n'ont pas eu les mêmes scrupules. Il s'agit ici d'un thème ancien, né dans le contexte de la séparation théologique entre le judaïsme et le christianisme naissant; le thème qui est au cœur du débat entre Origène et Héraclide dévoile ses racines judéo-chrétiennes toujours présentes dont malgré les transformations subies pendant presque deux siècles.

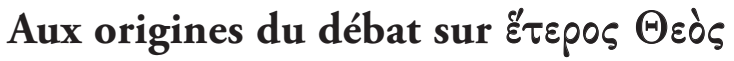

La question de «l'autre dieu» est très probablement née comme un thème apologétique judéo-chrétien qui a bien évolué à l'époque d'Origène. On le voit apparaître surtout dans le débat sur l'unicité de Dieu questionnée par la confession chrétienne de la foi en Christ «Fils de Dieu». Le premier à en parler est sans doute Justin Martyr. À la même époque, les Marcionites ont introduit

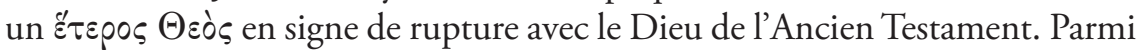
les textes qui abordent cette question, se trouvent les écrits de Justin Martyr (Dialogue avec Tryphon et l'Apologie) et le recueil des Homélies pseudo-clémentines ${ }^{55}$.

53. Cf. ComJoh I, 214

54. ComJoh I, 215; Blanc, SC 120bis, p. 164-165.

55. Justin Martyr parle de Simon le Magicien dans son Apologie, au même endroit où il présente Marcion: cf. Apologie 26.1-3. 
Ils permettent de situer les origines du débat qui se déroule dans l'Entretien avec Héraclide dans le sillage d'une controverse entre le judaïsme et le christianisme naissant, et ainsi ils nous renseignent sur les enjeux théologiques de

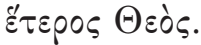

Les commentateurs notent surtout la présence de cette expression chez

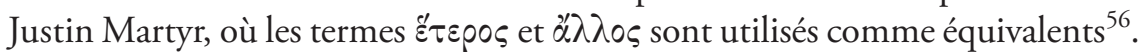
Dans l'Apologie se trouvent deux fragments qui illustrent cette équivalence et dans les deux cas il s'agit de la doctrine de Marcion:

Un certain Marcion, originaire du Pont, qui encore aujourd'hui instruit ses

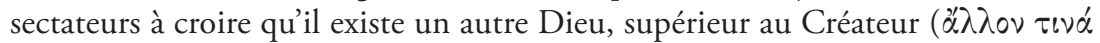
... $\theta \varepsilon o ́ v)$; avec l'assistance des démons il a mené un grand nombre de gens, à travers le monde entier, à proférer des blasphèmes et à renier le Dieu créateur de cet

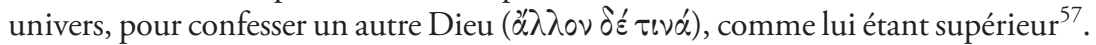
Les mauvais démons ont suscité aussi Marcion, originaire du Pont, comme nous l'avons dit plus haut, qui maintenant encore enseigne à nier que Dieu soit le créateur de tout ce qui existe au ciel et sur la terre et que celui qui a été annoncé

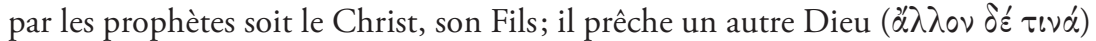
à côté du Dieu créateur de l'univers, et pareillement un autre fils (xai ópoí $\omega$ s

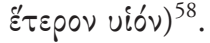

Ces deux passages confirment l'opinion selon laquelle «autre dieu» fait partie des idées marcionites. Plus intéressant est le débat sur cet "autre Dieu» dans le Dialogue avec Tryphon, car il concerne la relation entre le monothéisme et la possibilité de le réconcilier avec la divinité du Christ. Les emplois plus

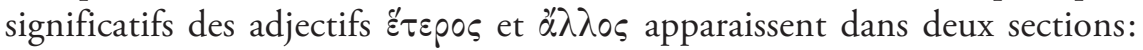
Dial 55-62 et 126-129. Cependant, dans les deux cas la question du rapport entre l'unicité de Dieu et la divinité du Christ reste primordiale.

Dialogue avec Tryphon aborde très vite la question de l'unicité de Dieu, lorsque Justin déclare: «Il ne saurait y avoir d'autre Dieu (ă $\left.\lambda \lambda \lambda_{0 \varsigma} \Theta \varepsilon o ̀ \varsigma\right)$, Tryphon, et il n'y en eut jamais de toute éternité, que Celui qui a créé et ordonné cet univers. ${ }^{59}$ Après cette mise au point concernant le monothéisme commun aux Juifs et aux chrétiens, la question de «l'autre Dieu» est discutée à partir des exemples tirés de l'Ancien Testament. L'invitation vient du juif Tryphon qui dans cette expression voit la négation du monothéisme des

56. Sur le rapport entre Justin et l'Entretien, $c f$. M.J.E. Edwards, Origen Against Plato, op. cit., p. 68, et p. 83. Même si une légère différence existe, les deux termes sont confondus à l'usage; $c f$. P. Henne, "Pour Justin, Jésus est-il un autre Dieu?», Revue des sciences philosophiques et théologiques, $\mathrm{n}^{\circ}$ 81, 1997, p. 57-58.

57. Apologie 26, 5; Munier, SC 507, p. 200-201.

58. Apologie 58, 1; Munier, SC 507, p.

59. Dial. 11, 1; Ph. Bobichon, Justin martyr. Dialogue avec Tryphon (éd. critique, trad., comment.), Fribourg, Academic Press, 2003, p. 210-211; p. 610-611, col. Paradosis. 
Écritures et le signe du culte païen. Il défie Justin de lui démontrer «que

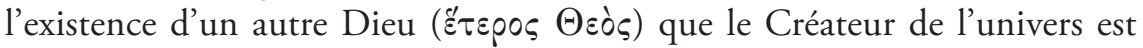
attestée par l'Esprit prophétique». Il refuse d'avance ce titre au soleil et à la lune, ainsi que le recours à l'expression "Dieu des dieux et Seigneur des seigneurs» (Deut 10, 17; Ps. 135, 2-3; Ps 95, 4; 1 Chron. 16, 25; Néh. 1, $5 ; 9.32)$. Ceux qui sont regardés comme dieux ne le sont pas en comparaison avec le Dieu véritable ( $c f$. Ps 95, 5; Ps 113, 12. 16). Sur ce point, Origène aurait été pleinement d'accord avec lui.

Pour éviter de débattre sur l'idolâtrie, Justin cite des théophanies, comme la visite des trois anges chez Abraham (Gen 18, 1-3; Gen. 19, 27-28). Et il continue:

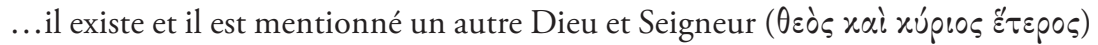
au-dessous du Créateur de l'univers; il est appelé ange parce qu'il annonce aux hommes tout ce que veut leur annoncer le Créateur de l'univers, au-dessus duquel

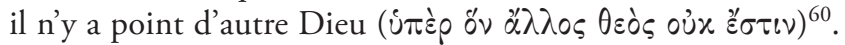

Après un court échange sur ces versets, Justin s'explique davantage:

...ce Dieu qui, selon ce qui y est dit et écrit, s'est fait voir à Abraham, à Jacob

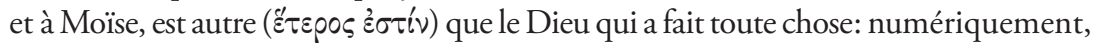
j'entends, et non pour la pensée. Car j'affirme qu'il n'a rien fait ni dit que ce que Celui qui a créé l'univers, au-dessus duquel il n'est point d'autre Dieu (i் $\pi \dot{e} \rho$ öv

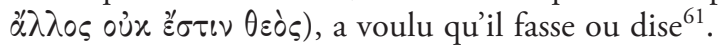

En partant de l'affirmation que personne n'a jamais vu le Dieu Yahvé, Justin rassemble les versets de l'Ancien Testament qui parlent d'un «Seigneur» ou d'un "dieu» visible ou manifesté aux hommes sous une forme sensible, voire même une forme humaine ${ }^{62}$. La dernière théophanie qui est discutée est celle sur le Sinaï, lorsque Moïse voit le buisson-ardent (Ex. 3, 2-4) qui l'amène à la conclusion que "celui qui, à la demande du Père et Seigneur, et en servant (ainsi) sa volonté, s'est fait voir à Abraham, à Isaac, à Jacob et aux autres patriarches, les Écritures le nomment Dieu» ${ }^{63}$. Cette argumentation

60. Dial. 56, 4; Bobichon, p. 324-325.

61. Dial 56, 11; Bobichon, p. 328-329. Le dialogue en $\$ 56$ annonce un échange plus élaboré dans les paragraphes qui suivent (\$\$ 56-61).

62. "...d'autres textes encore rapportent de quelle façon, lorsqu'il fuyait son frère Esau, s'est fait voir à celui qui est à la fois ange, Dieu, et Seigneur, qui sous forme d'un homme apparut à Abraham, et sous forme d'un être humain a combattu Jacob lui-même» Dial. 58, 10; Bobichon, p. 340-341. Dans Gen. 19, 20-25, le titre «Seigneur» est attribué également à un ange. Un «autre Dieu» s'est manifesté à Jacob (Gen. 31, 10-13; Gen. 32, 22-30; Gen. 35, 6-10).

63. Dial, 126, 5; Bobichon, p. 525. 
est extrêmement intéressante car elle permet de justifier, les Écritures à l'appui, la présence d'un Dieu autre que le Dieu Yahvé. Celui-ci serait sa forme visible, accessible aux hommes.

Aux demandes pressantes de Tryphon qui ne voit pas comment toute cette démonstration aurait pu concerner l'homme Jésus, Justin répond par d'autres versets où se trouvent les différents noms attribués à son envoyé: principe, avant toutes les créatures, gloire du Seigneur, fils, sagesse, ange, dieu, seigneur, verbe, etc ${ }^{64}$.

Dans l'argumentation de Justin Martyr, on reconnaît facilement certains éléments de celle d'Origène: il y a des "autres dieux» mentionnés dans l'Ancien Testament et la divinité du Christ se manifeste à travers une série de noms (ou caractéristiques) bibliques, relatifs au Dieu unique. Il y a toutefois des différences importantes: Justin fait un effort pour prouver à son interlocuteur que Jésus-Christ est «dieu» et cela en conformité avec les Écritures. Mais même dans cette démonstration, il se garde bien d'appeler le Christ «l'autre dieu», probablement à cause de l'utilisation de cette expression par les Marcionites.

\section{Quand Simon le Mage discute avec l'apôtre Pierre}

Le témoignage du recueil pseudo-clémentin (dont les dates de rédactions proposées se situent entre les $\mathrm{II}^{\mathrm{e}}$ et $\mathrm{IV}^{\mathrm{e}}$ siècles) doit être traité avec beaucoup de précautions. Tout d'abord, l'attribution de l'expression "autre dieu» à Simon le Mage est hautement problématique et on peut douter qu'il ait réellement prêché la distinction entre le Dieu de l'Ancien et du Nouveau Testament ${ }^{65}$. On suppose plutôt que seulement une petite partie de la doctrine exposée par Simon dans ce recueil est réellement de lui et que, en revanche, on lui fait

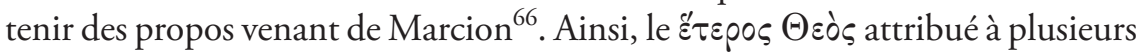

64. Dial 61, 1; Bobichon, p. 347.

65. Cf. S. Pétrement, Le Dieu séparé..., op. cit., p. 325-342. Sur le rapport entre cette description et la doctrine exprimée par Simon ailleurs, S. Haar, Simon Magus:the first gnostic?, W. de Gruyter, 2003, col. «Beihefte zur Zeitschrift für die Neutestamentlisch Wissenschaft», p. 109-112.

66. Sur l'origine simonienne d'une petite partie des propos, voir K. Beyschlag, Simon Magus und de christiliche Gnosis, Tübingen, Mohr, 1974, p. 64; G. Lüdemann, Untersuchungen sur simonianischen Gnosis, Göttingen, Vandenhoeck \& Ruprecht, 1975. L'idée que Simon le Mage soit un porte-parole de Marcion est discutable. Elle est soutenue par F.-M. Braun, "Marcion et la gnose simonienne», Byzantion, n' 25-26-27, 1955-1956-1957, p. 631-648 et G. Lüdemann, Paulus der Heidenapostel. II. Antipaulinismus in frühen Christentum, Göttingen, 1983, p. 228-248 contra A. Salles, "Simon le Magicien ou Marcion?», Vigiliae christianae, $\mathrm{n}^{\circ} 12,1958$, p. 197-224. 
reprises à Simon par l'auteur des Homélies pseudo-clémentines, s'inspire très probablement des thèses marcionites. Les échanges entre Simon et l'apôtre Pierre sont d'autant plus intéressants que les positions des protagonistes sont inversées par rapport à celles de Justin Martyr et du juif Tryphon. Dans les Homélies, c'est Simon qui soutien la possibilité de nommer le Christ «dieu» et "autre dieu», tandis que l'apôtre Pierre défend le monothéisme le plus strict, même s'il le fait avec des arguments différents de ceux de Tryphon.

Dans l'homélie III, Pierre caricature la doctrine prêchée par Simon et l'incite à démontrer - à partir des Écritures - que...

...celui qui a fait le ciel et la terre, et tout ce que s'y trouve, n'est pas le Dieu suprême, mais qu'il existe un autre, inconnu et suprême ( $\alpha^{\prime} \lambda \lambda \circ \nu \tau \iota \nu \dot{\alpha} \alpha{ }^{\prime} \gamma \nu \omega \sigma \tau o \nu$ $\tau \iota \nu \dot{\alpha} \alpha \nu \omega ́ \tau \alpha \tau o \nu)$, comme étant d'une manière indicible Dieu des dieux, et que

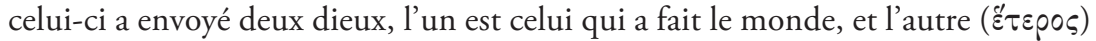
celui qui a donné la loi ${ }^{67}$.

Cette promesse est réalisée dans l'homélie XVI qui a pour objet l'unité de Dieu. Le débat commence par le rapport entre le Dieu unique et les dieux dont parlent les Écritures, pour transposer cette problématique sur le terrain de la christologie. Voici comment les deux protagonistes présentent leurs positions respectives:

Pierre prit alors la parole: «Pour moi, déclara-t-il... Je soutiens qu'il n’y a qu'un seul Dieu, le créateur du ciel et de la terre avec tout ce qu'ils renferment. Il n'est

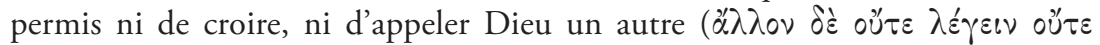

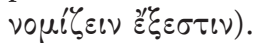

— Et moi, dit Simon, je soutiens que les Écritures qui sont en crédit chez les Juifs parlent de plusieurs dieux ( $\pi 0 \lambda \lambda$ où $\theta \varepsilon \circ u ́ \varsigma)$, et que Dieu ne s'irrite pas de cela, puisque c'est lui-même qui déclare par ses Écritures, qu'il y a plusieurs $\operatorname{dieux}^{68}$.

Dans un long passage attribué à Simon le Magicien, sont mentionnés certains versets de l'Ancien Testament où il est question des "dieux" ${ }^{69}$. En répondant à cette démonstration, l'apôtre Pierre affirme la supériorité du «Dieu

67. Hom Clem 3, 2; trad. A. Siouville, p. 129; texte grec: Die Pseudoklementinen I Homilien, B. Rehm, G. Strecker (éd.), GCS, Berlin, Akademie Verlag, 1992, p. 57.

68. HomClem 16, 5; p. 307; texte gr. Die Pseudoklementinen, B. Rehm (éd.), coll. "Die griechischen christlichen Schriftsteller der ersten Jahrhunderte», $\mathrm{n}^{\circ} 42$, Leipzig, 1953, p. 220.

69. Signalons qu'il s'agit de fragments différents de ceux qui sont utilisés par Justin Martyr; cf. Gen. 3, 21 «'homme est devenu comme l'un de nous»; Ex 22, 28; Deut. 4, 34; Jér. 10, 11; Deut. 13, 6; 10, 14. 17-18; Ps. 86 (85), 8; Ps 50 (49), 1; Ps 82 (81), 1; Deut. 4, 35. 39; 6, 4. 13; cf. Hom Clem 16, 6; p. 308-309. 
des Juifs» et cite les versets qui disent «il n'y a pas d'autre Dieu», dans lesquels

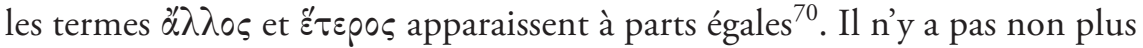

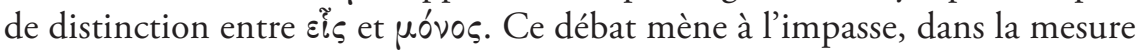
où il porte sur la lettre de la formule et qu'il n'est pas accompagné de la moindre réflexion sur la nature de cette différence. Les protagonistes doivent passer à des interprétations plus serrées. Ils prennent alors comme exemple le verset Gen 1, 26 "Faisons l'homme à notre image et notre ressemblance», et plus précisément le pluriel «faisons». Est-il un signe de la présence d'un autre Créateur? Ou de plusieurs? Contre Simon - qui voit ici un témoignage

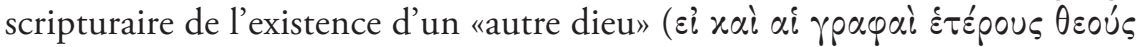

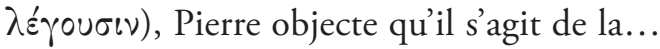

...sagesse... celle avec laquelle Dieu lui-même se réjouissait sans cesse comme

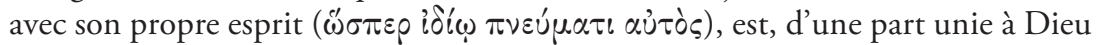
comme une âme, et, d'autre part, s'étend hors de lui, comme une main, créant l'univers ${ }^{71}$.

Pour éviter de parler d'une "puissance» ou d'un «dieu», Pierre compare la sagesse à l'esprit ou à l'âme du Créateur. Il poursuit cette logique en faisant allusion aux versets concernant l'origine du premier couple (Gen 2, 21-22):

C'est pour cela qu'il n'a été fait qu'un seul homme et que le sexe féminin est sorti

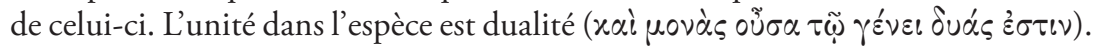
C'est en effet selon l'extension et la contraction que l'unité est considérée comme

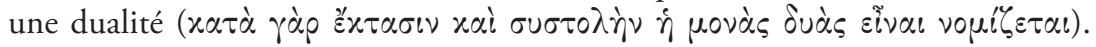

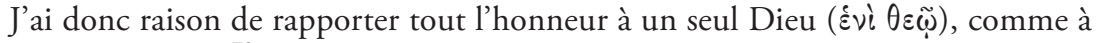
des père et mère ${ }^{72}$.

Autrement dit, selon Pierre, «Dieu» étant unique contient en lui une dualité, voire une pluralité d'êtres. Ainsi les «dieux» dont parlent les Écritures ne sont rien d'autre que les «extensions» de l'unique être divin. Cette proposition va revenir quelques paragraphes plus loin, après que le débat aborde la question traditionnelle de l'opposition entre le Dieu unique des chrétiens et les nombreux faux dieux des païens - c'est ainsi que l'apôtre tente d'abolir les arguments scripturaires de Simon, en utilisant surtout les citations des livres prophétiques ( $c f .16,13)$.

Léchange devient plus serré lorsque Simon riposte en évoquant le Christ il a été mis à mort avec raison, car il s'est proclamé «Fils de Dieu». Avec ce

70. Les passages provenant principalement des livres prophétiques: Jos. 23, 7; Ex. 22, 28; Jér. 10, 11; Gen 1, 1; Ps 19 (18), 2; Ps. 102 (101), 27-28: HomClem 16, 7; p. 309-310.

71. Hom Clem 16, 12; p. 312; texte grec, p. 223-224.

72. Hom Clem 16, 12; p. 312; texte grec, p. 224 


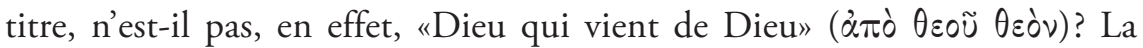
réponse de l'apôtre est pour le moins consternante pour le lecteur moderne, car il refuse d'attribuer l'appellation «dieu» à Jésus et souligne sa soumission filiale à Dieu:

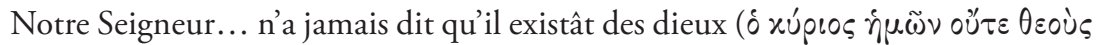

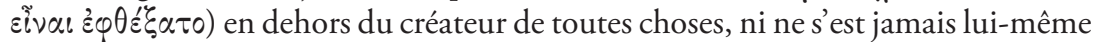

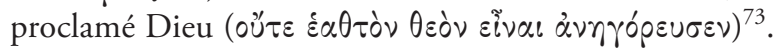

Ensuite, il évoque les âmes humaines:

les corps des hommes renferment des âmes immortelles, revêtues du souffle de

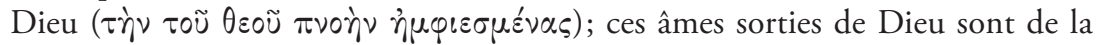

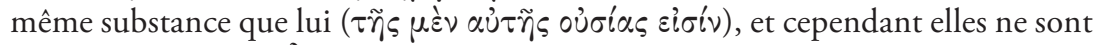

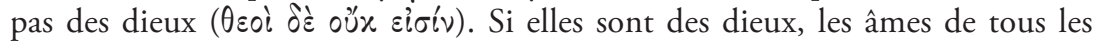
hommes, morts, vivants ou encore à naître, se trouvent être, d'après cette manière

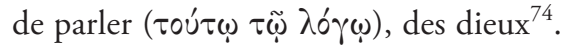

La conclusion de l'apôtre Pierre est particulièrement troublante: si on peut dire que les âmes sont des dieux, alors le Christ peut l'être aussi.

La question de «l'autre dieu» est évoquée au début de l'homélie XVIII où Simon le Magicien revient à la charge en disant:

....je t'ai promis de revenir aujourd'hui pour discuter avec toi et te prouver que le créateur du monde n'est pas le Dieu suprême, mais celui-ci est un autre Dieu,

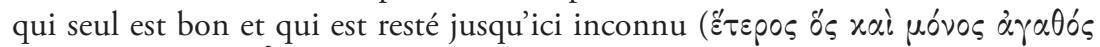

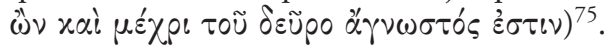

Cependant, le débat change bien par rapport à ce que nous avons vu jusqu’à présent. Simon le Magicien expose l'idée de «Dieu inconnu» (homélie XVIII) et Dieu - Créateur responsable du mal. Évidemment, Simon ne donne pas au «Mauvais» le statut de «l'autre Dieu». Son «autre Dieu» est le Dieu bon, le Dieu inconnu, celui qui n'est pas et le Créateur de la matière est l'auteur du mal.

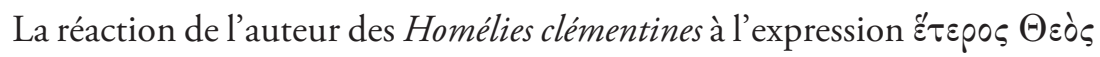
est très significative. L'apôtre Pierre, représentant par excellence de l'enseignement apostolique, expose une théologie fortement imprégnée du judéo-christianisme, focalisée sur l'unicité de Dieu au point d'avoir des difficultés à formuler l'idée de la divinité du Christ ${ }^{76}$. Or, cette position est proche de celle d'Héraclide.

73. Hom Clem 16, 15; texte grec, p. 225.

74. Hom Clem 16, 16; p. 314; texte grec, p. 225-226.

75. Hom Clem 18, 1; p. 332; texte grec p. 241.

76. Cf. Introduction, p. 36-37. 
En effet, les fragments de l'homélie XVI rappellent les passages de l'Entretien

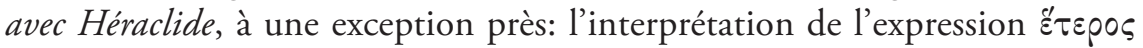
Osòs. Notons surtout l'utilisation d'exemples semblables. Dans les deux cas, on se réfere au premier couple humain comme modèle de l'unité des êtres réciproquement différents, «autres», dont l'unité renvoie à celle de Dieu. Pareil pour le second exemple, concernant l'unité entre l'homme et Dieu. Origène montre comment cette unité se réalise dans l'esprit $(\pi \nu \varepsilon u ̈ \mu \alpha)$ qui vient de Dieu. Son exemple est une correction de l'argument de Pierre qui parle des âmes humaines enveloppées dans un souffle $(\pi v \circ \grave{\eta})$ divin. En ajoutant les Homélies clémentines au dossier, nous pouvons ainsi retracer les

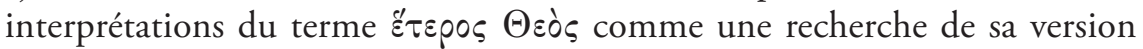
orthodoxe, forgée au cours du débat sur le monothéisme, le polythéisme et le dualisme.

\section{Conclusion}

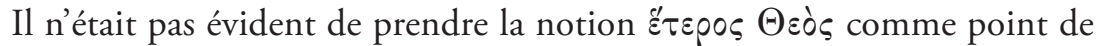
départ pour aborder la question du dualisme chez Origène. Pourtant, elle a permis d'approcher cette problématique, extrêmement complexe, et de la situer au carrefour des thèmes habituellement traités séparément, tels que la théologie trinitaire, la christologie, l'angélologie, les polémiques contre le polythéisme païen et contre le monothéisme juif. Car, en effet, le dualisme est une idée transversale qui peut surgir dans des domaines différents.

Une deuxième remarque concerne le vocabulaire. Parler de «l'autre Dieu» ne dénote pas une position forcément dualiste, mais constitue l'indice d'un dualisme potentiel. La différence peut s'exprimer de multiples manières, mais seulement certaines d'entre elles expriment une pensée dualiste. Les exemples étudiés plus haut montrent bien ces ambiguïtés liées au concept de différence et donc aussi dans la manière même de concevoir le dualisme. Indépendamment de la définition de l'orthodoxie et de l'appartenance, la même notion عُ

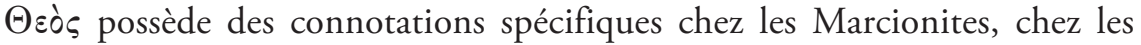
Monarchianistes, chez les Juifs et chez les judéo-chrétiens, et enfin chez Origène. 